\section{Parts and the basic level in natural categories and artificial stimuli: Comments on Murphy (1991)}

\author{
BARBARA TVERSKY \\ Stanford University, Stanford, California \\ and \\ KATHLEEN HEMENWAY \\ Sun Microsystems, Mountain View, California
}

\begin{abstract}
Natural taxonomies consist of categories that vary in level of abstraction. Categories at the basic level, such as chair and ap. ple, are preferred in a broad range of situations (Rosch, Mervis, Gray, Johnson, \& Boyes-Braem, 1976). Several studies have rev ealed qualitative differences between the basic level and other levels. For example, Tuersky and Hemenway (1984) presented evidence that parts proliferate at the basic level; they proposed that parts link the appearance of category members with their functions. Although not taking issue with these findings, Murphy (1991) investigated whether parts are necessary or sufficient for a basic level In an attempt to demonstrate that parts are not necessary, Murphy used artificial stimuli that did not capture the essential features of natural taxonomies. These discrepancies preclude any conclusions based on his studies. Murphy's data also do not support his claim that parts are not sufficient for a basic level Finally, it is unlikely that pursuing questions of neces sity or sufficiency will produce insights into human categorization.
\end{abstract}

The world presents us with an uncountable number of different things. One way people cope with these numbers and make order out of them as well is to group similar things together into categories, and categories into taxonomies. Thus, there are furniture, chairs, and kitchen chairs, and fruit, apples, and pippin apples. Intriguingly, although the same thing, a kitchen chair or a pippin apple, can be referred to by labels at all those levels, a single level of abstraction is preferred by most people in a wide variety of situations (Brown, 1958; Rosch et al., 1976). That level-the level of chair and apple-has been termed the basic level (Berlin, Breedlove, \& Raven, 1973; Rosch et al., 1976).

Categories not only reduce the number of distinctions that must be kept in mind, they are also informative. For example, if you know that a pomelo is a fruit, you can infer that it is edible, grows on trees, and so forth. If you know that it is a citrus fruit, you can infer even more features. Thus, the more specific a category, the more informative. However, there is a cost to the informativeness: The more informative a category is, the more distinctions you must keep in mind. More specific cate-

Preparation of this manuscript was facilitated by the Air Force Office of Scientific Research, Air Force Systems Command, USAF, under Grant AFOSR 89-0076 to Stanford University. We are grateful to Karen Ravn for helpful discussion. Send correspondence to Barbara Tversky. Department of Psychology, Building 420, Stanford University, Stan. ford, CA $94305-2130$ gories have more contrast categories than do less specific categories.

Rosch and her collaborators (Rosch, 1978; Rosch et al., 1976) argued persuasively that the basic level maximized one conception of cognitive economy-it is relatively more informative at a relatively lower cost. Rosch et al. indexed informativeness by the number of attributes students listed for categories at three levels of abstraction. They indexed cost by the number of categories or distinctions that had to be kept in mind. Specifically, students listed only a couple of attributes for superordinate (object) categories, whereas they listed several times that number for basiclevel categories and only a few additional attributes for subordinate categories. At the basic level, categories have lots of attributes while having relatively few contrast categories, which makes them informative at relatively low cost.

Rosch and her collaborators went on to demonstrate a large number of cognitive tasks that converged on the basic level. Some of those tasks depended on the appearance of the objects, whereas others depended on function or on language. For example, appearance-dependent tasks showed that the basic level is the highest level at which subjects can recognize composite outline drawings. Function-dependent tasks showed that the basic level is the highest level for which behavior toward exemplars is similar. Language-dependent tasks showed that the basic-level label is preferred in naming pictures.

Whereas Rosch and her collaborators emphasized the quantitative differences between the levels, later research revealed qualitative differences. For example, in the attributes collected by Rosch and her collaborators, superordinate categories were typically characterized by functional features, such as "you wear it" for clothing and "[you use it to] make things" and "[you use it to] fix things" for tools. In contrast, basic and subordinate categories elicited many features that could be perceived in addition to functions.

For a broad range of natural categories, including both objects and organisms, we (Tversky \& Hemenway, 1984) found that one kind of feature proliferates at the basic level, namely, parts, such as the handle and blade of a knife and the peel and pulp of a banana. We suggested that parts have a functional aspect as well as a perceptual aspect. For example, the handle of a knife is used for grasping and the blade for cutting. The peel of a banana protects the pulp and is disposed of, and the pulp is eaten. We speculated that parts form a bridge between the appearance, on the one hand, and the function or behavior of objects and organisms, on the other. Parts make basic concepts informative by allowing inferences from appearance to function.

These insights about the basic level of categorization and many other insights as well were achieved by research using natural categories. It is hard to imagine that phe- 
nomena such as the relationship between relative informativeness and the basic level, or that between family resemblance structure and typicality, or that between the basic level and parts would have been discovered using artificial stimuli. The multitude of things the world contains can be categorized in many different ways according to many different criteria. The apparent universality in the kinds of categories people form-including that common basic categories are characterized by partsreveals a great deal about human cognition.

In his paper, Murphy (1991) did not take issue either with the findings that part attributes are diagnostic of the basic level across a broad range of natural categories or with the speculations about the role of parts in relating appearance to function and in associated inferences. Rather, he asked if parts are necessary or sufficient for a basic level, questions unrelated to our claims and speculations. Here, we will argue that Murphy's case that parts are neither necessary nor sufficient for the basic level is not substantiated by his data and also that pursuing questions of necessity and sufficiency is unlikely to produce insights into human categorization. But first we will review Murphy's findings.

To investigate the necessity and sufficiency of parts, Murphy used two sets of artificial visual stimuli with nonsense labels. The stimuli formed hierarchies in which the quantitative pattern of visual features to some extent mimicked that found in natural categories. The first set of stimuli were intended to have no parts; the second set, to have parts as well as other visual attributes. Drawing conclusions about categorization from artificial stimuli is no simple matter, especially for the basic level (see Brown, 1980, and Lassaline, Wisniewski, \& Medin, 1991, for critiques similar to our own). The artificial stimuli must adequately model the structure and features of natural categories and taxonomies, and the experimental tasks must be unconfounded. Murphy said that he resorted to the use of artificial stimuli because "it was impossible to find actual category taxonomies that did not have parts collected at the basic level" (p. 436). This in itself is informative, since many of the taxonomies that have been studied were of abstract categories, such as events.

Murphy's research program had two parts, each based on one of the two sets of stimuli. In the first part, he claimed to demonstrate that for the visual stimuli that purportedly had no parts, the middle level was basic according to two criteria: Verifying the middle-level label was faster than verifying the highest or lowest level labels, and the ratio of within-category to between-category similarity was highest for the middle level. This led Murphy to conclude that parts are not necessary for a basic level (Experiments 1-3). In the second part, he used visual stimuli with both part and nonpart features that had previously exhibited a basic level according to the relativelabeling-time criterion. He found that adding other visual attributes to the middle level increased the difference in verification times between the middle and lowest levels (Experiment 4) and that adding other visual attributes to the highest level eliminated differences in verification time between the middle and highest levels (Experiment 5), leading Murphy to conclude that parts are not sufficient for a basic level.

Although Murphy's findings and conclusions in no way contradict our own (Tversky \& Hemenway, 1984), we find them unsupported and misleading. Briefly, the "parts are not necessary for a basic level" conclusion was based on studies using an artificial taxonomy that did not capture enough essential properties of natural category taxonomies to justify conclusions about a basic level. And the "parts are not sufficient for a basic level" conclusion was, on closer inspection, not supported by the data. We consider each set of studies in more detail.

The stimuli of Experiments 1-3 were rectangular forms that bore a strong resemblance to postage stamps and that varied in color at the highest level and in markings on borders and insides and in size at the middle and lowest levels. These stimuli had no functions, either self-evident or given. They were perceptually defined at all levels of abstraction. Thus, they violate the qualitative pattern of features in natural categories, in which for superordinate categories, functional features outnumber perceptual ones, even for organisms, and functional features appear at lower levels as well. Moreover, all of the stimuli at all levels had the same general shape, despite numerous findings that shape is a property shared by different subordinates belonging to the same basic-level category and that different basic-level objects have different shapes. Thus, if any analogy to natural taxonomies holds, it would be that the highest level of these stimuli are comparable to subordinate categories. Given that these stimuli do not capture the essential features of natural superordinate, basic, and subordinate categories, it is not appropriate to draw conclusions about the basic level from research on them.

Although the failure of these stimuli to incorporate the essential features of natural taxonomies precludes drawing any conclusions about the basic level, there were other problems with the stimuli. The stimuli were designed to have no parts, but they had internal markings comparable to the stripes of a zebra or the spots of a leopard, which have been called parts by informants in other studies. Murphy attempted to avert criticism on this account by collecting part judgments from one set of informants (Experiment 1B) and attribute listings from another set of informants (Experiment 1A). He did not adopt the practice of previous research of giving the attribute listings to a set of informants to judge which of these were parts (parts are, after all, a subset of attributes). Moreover, the procedure for eliciting parts was inexplicably different from that of eliciting attributes. He did find that the relative increase in number of parts from the highest to the middle level was greater than the increase from the middle to the lowest level, as in Tversky and Hemenway (1984), but the absolute numbers of parts reported was relatively small, especially compared with the number of attributes elicited. However, the number of attributes 
listed was surprisingly high, nearly as high as for natural categories, which have a rich and familiar set of attributes, and far higher than the number of attributes Murphy used to describe the stimuli. It is possible that the unusual procedures used to elicit attributes and parts led to the unexpectedly large number of attributes and the relatively small number of parts.

The final two experiments used the tool-like stimuli of Murphy and Smith (1982). Unlike the first set, they were functionally as well as perceptually defined at the highest level. The highest level categories, "pounders" and "cutters," had parts as well as other features and had functions that were apparent from their parts. These stimuli appear to mirror more of the characteristic features of natural taxonomies than the first set. Pounders and cutters correspond to a level between the established basic level, hammer, and the natural superordinate, tool. This level is comparable to the categories "string instrument" and "wind instrument" as subcategories of "musical instrument." To test whether parts were sufficient for a basic level, Murphy added nonpart visual features to the middle and highest levels, respectively. He reasoned that if nonpart features could shift the basic level, then parts are not sufficient for a basic level.

In Experiment 4, nonpart visual features-colors and dots or stripes-were added to the different lower-level stimuli belonging to a particular middle-level category. This was the "enhanced" condition in contrast to the "simple" condition. This is analogous to making all chairs green with red stripes and all tables blue with yellow polka dots. Not surprisingly, it took longer to label stimuli at the lowest level in the enhanced condition than in the simple condition, just as in the example it would take longer to discriminate armchairs from kitchen chairs if they were all green with red stripes and to discriminate kitchen tables from dining-room tables if they were all blue with yellow dots. Although Murphy noted in support of his claim that the difference in labeling times between the lower and middle levels was greater for the enhanced condition, this follows from the fact that times for the lowest level increased. There were no differences between the simple and enhanced conditions in reaction times to the middle levels or in the difference between the middle and highest levels. From this pattern of data, increased labeling time at the lowest level, one cannot infer, as Murphy did, that the "basic" level has been enhanced. One can only infer that the lowest level has been made more difficult, and that is readily explained by decreased discriminability.

In Experiment 5, nonpart visual features were added to the highest level stimuli, in particular, color, size, continuous or broken border, and dots or stripes. The aim was to eliminate a basic-level effect. This is analogous to making all fruits large, blue with yellow polka dots, and with continuous borders, and all vegetables small, green with red stripes, and with discontinuous borders. The consequence of the added features is to make the two highest level groups more discriminable and to make the middle-level categories less discriminable. With these stimuli, labeling times to the highest level did not differ from labeling times to the middle level, although both were faster than labeling times to the lowest level, an effect accounted for by the change in discriminability. But "no difference" is not the same as eliminating a basic level, Murphy's conclusion from these data. Moreover, adding a large number of visual features to the highest level again violates the structure of natural category taxonomies. Natural superordinate categories have very few features, and for object categories, these are almost always functional and not perceptual in character.

Thus, despite Murphy's claims, neither of these experiments has provided evidence for a shift in the basic level. If anything, these data attest to the robustness of the basic level. Murphy's findings were caused by adding visual features to middle- or highest level stimuli and are readily accounted for by changes in the discriminability of the visual forms. There are many factors that increase or decrease labeling times. One of these is discriminability of stimuli. Another is frequency or familiarity of labels. These factors, and others, can affect labeling time without having any effect on levels of categorization.

To summarize so far, we have shown that Murphy's claim that parts are not necessary for a basic level was not supported because that claim was based on artificial stimuli that did not incorporate the essential properties of natural category taxonomies. The claim that parts are not sufficient for a basic level was not supported for several reasons, including that the data were not sufficiently strong, that the data can be accounted for in terms of changes in discriminability, and that the addition of perceptual features to the highest level violates the structure of natural categories.

It is puzzling to pursue questions of necessity and sufficiency in categorization, given that they are most likely unanswerable. Years of investigations into the nature of categories have led to a widely held consensus that it is difficult, if not impossible, to discover sets of necessary or sufficient features for category membership in natural categories (e.g. . Putnam, 1975; Wittgenstein, 1958). And whether or not there are such conditions for category membership, it is abundantly apparent that people's use of categories reflects other principles, such as typicality, rather than necessary and sufficient conditions (see, e.g., Armstrong, Gleitman, \& Gleitman, 1983; Lakoff, 1987; Rosch, 1978; Rosch \& Mervis, 1975; Smith \& Medin, 1981). Given that the search for necessary and sufficient conditions of category membership for natural categories has proved to be not only fruitless but, more significantly, irrelevant to characterizing human categorization, one could hardly encourage a quest to find necessary and sufficient conditions for a second- or third-order phenomenon like the basic level.

In contrast to the quest for necessary and sufficient features for categories, modern research on natural categories has provided many insights from the study of the sorts of categories people naturally form and use in their ordi- 
nary lives. These categories have a basic level, and it is characterized by parts.

\section{REFERENCES}

Armstrong, S. L., Gleitman, L. R., \& Gleitman, H. (1983). What some concepts might not be. Cognition, 13, 263-308.

BerLiN, B., Breedlove, D. E., \& RAveN, P. H. (1973). General principles of classification and nomenclature in folk biology. American Anthropologist, 75, 214-242.

Brown, R. (1958). How shall a thing be called? Psychological Review, 65, 14-21.

Brown, R. (1980). Natural categories and basic objects in the domain of persons. Katz-Newcomb Lecture, University of Michigan, Ann Arbor, MI.

LAKOFF, G. (1987). Women, fire and dangerous things: What categories reveal about the mind. Chicago: University of Chicago Press.

Lassaline, M. E., Wisniewski, E. J., Medin, D. L. (1991). The basic level in artificial and natural categories: Are all basic levels created equal? In B. Burns (Ed.), Percepts, concepts and categories: The representation and processing of information. Amsterdam: Elsevier.

MURPHY, G. L. (1991). Parts in object concepts: Experiments with artificial categories. Memory \& Cognition, 19, 423-438.
Murphy, G. L., \& Sith, E. E. (1982). Basic-level superiority in picrure categorization. Journal of Verbal Learning \& Verbal Behavior, 21, 1-20.

Putnam, H. (1975). The meaning of "meaning." In K. Gunderson (Ed.), Language, mind, and knowledge (pp. 215-271). Minneapolis: University of Minnesota Press.

Rosch, E. (1978). Principles of categorization. In E. Rosch \& B. B. Lloyd (Eds.), Cognition and categorization (pp. 27-48). Hillsdale, NJ: Erlbaum.

Rosch, E., \& Mervis, C. B. (1975). Family resemblances: Studies in the internal structure of categories. Cognitive Psychology, 7, 573-605.

Rosch, E., Mervis, C. B., Gray, W., Johnson, D., BoyesBraem, P. (1976). Basic objects in natural categories. Cognitive Psychology, 8, 382-439.

Smith, E. E., Medin, D. L. (1981). Categories and concepts. Cambridge: Harvard University Press.

TVersky, B., \& HemenWAY, K. (1984). Objects, parts, and categories. Journal of Experimental Psychology: General, 113, 169-193.

Wittgenstein, L. (1958). Philosophical investigations. Oxford: Blackwell.

(Manuscript received April 12, 1991; accepted for publication May 29, 1991.) 